Proc. Indian Acad. Sci. (Chem. Sci.), Vol. 102, No. 5, October 1990, pp. 585-592.

(C) Printed in India.

\title{
Effect of doping on stimulated Brillouin scattering in semiconductors
}

\author{
P AGHAMKAR* and P K SEN \\ Department of Physics, Barkatullah University, Bhopal 462 026, India
}

\begin{abstract}
Ahstract. We investigate stimulated Brillouin scattering (SBS) in doped nearly centrosymmetric $n$-type crystals like $n$-InSb and $n$-GaAs irradiated by pulsed high power lasers. The origin of the nonlinear interaction is considered to be in the third-order optical susceptibility arising due to both induced nonlinear current density and electrostriction. Using electromagnetic treatment, we have obtained the threshold condition for the onset of SBS and the effective Brillouin susceptibility $\chi_{\mathrm{B}, \text { eff }}$, as well as the resulting gain constant $\left(g_{\theta}\right)_{\text {eff }}$ for both lightly and heavily doped semiconductors. The detailed numerical analyses carried out favour heavily doped crystals to achieve high gain in the Stokes' component of the scattered electromagnetic mode. The analyses support the possibility of occurrence of optical phase conjugation via stimulated Brillouin scattering in selected doped semiconductors.

Numerical estimates made for a specific crystal $n$-InSb at $77 \mathrm{~K}$ using a pulsed $10.6 \mu \mathrm{m} \mathrm{CO}_{2}$ laser, establish its potential as candidate material for fabrication of phase conjugate mirrors when the crystal is heavily doped.
\end{abstract}

Keywords. Doped semiconductors; stimulated Brillouin scattering; $n$-type crystals; nonlinear interaction; optical susceptibility.

\section{Introduction}

When an intense light beam (pump wave) interacts with an active medium, strong optical amplification of the scattered wave occurs at Stokes' shifted frequency. Such phase-coherent processes are called stimulated scattering (SS) processes. Amongst these, the study of stimulated Raman scattering and stimulated Brillouin scattering (SRS and SBS) in solids has been the subject of intensive investigations (both theoretical and experimental) as these have manifold technological applications and provide understanding of the radiation-matter interactions in quantum electronics. An important current application of these processes in modern optics is to generate optical phase conjugation (OPC) in an active medium (Zel'dovich et al 1985).

When compared to other SS processes in solids, SBS may be regarded as a special case of SRS that involves acoustical phonons instead of optical phonons (Shen and Bloembergen 1965). SBS is most commonly used in producing OPC because of its purity and high conversion efficiency. Recently, Sen and Sen (1985) have established a correlation between SRS and SBS under the transient regime in weakly noncentrosymmetric solids. It is well known that the origin of SBS lies in the third-order nonlinear optical susceptibility of the medium. In all crystalline solids, the phenomenon of SBS arises due to a parametric coupling between the pump and the acoustic wave under finite electrostriction of the medium.

\footnotetext{
* For correspondence
} 
The present article deals with an analytical investigation of SBS in doped nearly-centrosymmetric $n$-type semiconducting crystals duly irradiated by single mode pulsed high power lasers. Due to the existence of sophisticated fabrication technology and the experimental observation of large optical nonlinearities in the vicinity of band-gap resonant transitions, semiconductors have been the natural choice as active media for the study of nonlinear optical phenomena, in preference to materials like gases and liquids (Jain 1982; Smith 1985). Direct-gap crystals like GaAs and InSb may be doped heavily with impurities to form high density electron gases (viz., $n_{0} \sim 10^{24}$ to $10^{25} \mathrm{~m}^{-3}$ ) (Mahan 1981). Moreover, variation in the doping level can enable one to study the collective effects (e.g., SRS,SBS, etc.) in solid-state plasma with electron plasma frequency spreading over the entire infrared spectrum (Hayes and Loudon 1978).

The present analysis of SBS is based upon the parametric coupling of three waves and the electrostrictive strain of the medium. The nonlinearities taken into account in the analysis are the nonlinear current density and the nonlinear electrostrictive polarization. The results manifest that the threshold pump field necessary for the onset of SBS increases with rise in the impurity doping level. The Brillouin gain constant well above the threshold can yield significant optical phase conjugation conversion efficiency only at a high doping level. Numerical estimations have been made for an $n$-type InSb crystal duly irradiated $10 \cdot 6 \mu \mathrm{m} \mathrm{CO}$ laser.

\section{Theoretical formulations}

We consider the lattice displacement of the crystals under the one-dimensional configuration. The basic equations employed in the present analytical investigation of SBS are:

$$
\begin{aligned}
& \frac{\partial^{2} u}{\partial t^{2}}-\frac{C \partial^{2} u}{\rho} \frac{\partial x^{2}}{2}+2 \Gamma_{a} \frac{\partial u}{\partial t}=\frac{r}{2 \rho} \frac{\partial}{\partial x}\left(E_{0} E_{1}^{*}\right), \\
& \frac{\partial v_{0}}{\partial t}+v v_{0}=\frac{e E_{0}}{m} \\
& \frac{\partial v_{1}}{\partial t}+v v_{1}+v_{0} \frac{\partial v_{1}}{\partial x}=\frac{e E_{1}}{m} \\
& v_{0} \frac{\partial n_{1}}{\partial x}+n_{0} \frac{\partial v_{1}}{\partial x}=-\frac{\partial n_{1}}{\partial t} \\
& P_{e s}=-r E_{0} \frac{\partial u^{*}}{\partial x}, \\
& \frac{\partial E_{1}}{\partial x}=\frac{n_{1} e}{\varepsilon}+\frac{r E_{0}}{\varepsilon} \frac{\partial^{2} u^{*}}{\partial x^{2}} .
\end{aligned}
$$

Equation (1) represents the motion of a lattice in the centrosymmetric crystals with $u, C, \rho, \Gamma_{a}$ and $r$ being the lattice displacement, elastic constant, uniform mass density, phenomenological acoustic damping parameter and electrostriction coefficient of the crystal, respectively. The driving term in the right hand side of equation (1) has its 
origin in the electrostrictive force induced by the pump electric field $E(t)=\mathrm{E}_{0} \cos \omega_{0} t$ via the process of electrostriction. Equations (2) and (3) are the zeroth and first-order momentum transfer equations with $v_{0}$ and $v_{1}$ being the zeroth and first-order oscillatory fluid velocities of the electron of effective mass $m$ and charge $e$, respectively. $v$ is the electron collision frequency. The electron continuity equation is given by (4). Equation (5) shows that the acoustic wave generated due to the electrostrictive strain modulates the dielectric constant and gives rise to nonlinear induced polarization $\boldsymbol{P}_{\text {es }}$. The space charge field $E_{1}$ is determined by the Poisson's equation (6), where $\varepsilon$ is the dielectric function of the semiconductor expressed as $\varepsilon_{0} \varepsilon_{1}, \varepsilon_{0}$ and $\varepsilon_{1}$ being the absolute permittivity and static dielectric constant of the crystal, respectively. $n_{1}$ is the first-order perturbation on the equilibrium electron concentration $n_{0}$ such that $n=n_{0}+n_{1}$.

Using (1) through (6), one can obtain on mathematical simplification,

$$
\frac{\partial^{2} n_{1}}{\partial t^{2}}+v \frac{\partial n_{1}}{\partial t}+\omega_{p}^{2} n_{1}-\frac{n_{0} r k^{2}}{\varepsilon} \bar{E} u^{*}=-\bar{E} \frac{\partial n_{1}}{\partial x}
$$

with $\bar{E}=e E(t) / m$ (say). We have neglected the Doppler shift $k v_{0}$ under the assumption that $\omega_{0} \gg v \gg k v_{0} . \omega_{p}$ is the electron plasma frequency given by $\omega_{p}=\left(n_{0} e^{2} / m \varepsilon\right)^{\frac{1}{2}}$. Here, we have considered the low-frequency perturbations of $v_{1}$ and $n_{1}$ to be proportional to $\exp \left[i\left(k_{a} x-\omega_{a} t\right)\right]$ characterizing the acoustic wave propagation. $n_{0}$ and $v_{0}$ are assumed to vary as $\exp \left(-i \omega_{0} t\right)$ under the spatially uniform pump field propagation regime. The density perturbation $n_{1}$ will have two components, viz. slow $\left(n_{1 s}\right)$ and fast $\left(n_{1 f}\right)$ ones, oscillating at frequencies $\omega_{a}$ and $\omega_{1}$, respectively. We have considered only the Stokes' components of the scattered electromagnetic wave (i.e. $\omega_{1}=\omega_{0}-\omega_{a}$ and $\mathbf{k}_{1}=\mathbf{k}_{0}-\mathbf{k}_{\mathbf{a}}$ ). One may assure oneself that the stimulated Brillouin process under consideration does not violate the phase matching conditions, viz., the energy and momentum conservation equations given by $\hbar \omega_{0}=\hbar \omega_{1}+\hbar \omega_{a}$ and $\hbar \mathbf{k}_{0}=\hbar \mathbf{k}_{1}+\hbar \mathbf{k}_{a}$. For spatially uniform laser radiation $\left|\mathbf{k}_{0}\right| \simeq 0$ yields $\left|\mathbf{k}_{1}\right| \simeq\left|\mathbf{k}_{a}\right|=k$ (say). Under rotating-wave approximation, (7) leads to the following coupled equations

$$
\frac{\partial^{2} n_{1 s}}{\partial t^{2}}+v \frac{\partial n_{1 s}}{\partial t}+\omega_{p}^{2} n_{1 s}=-\bar{E} \frac{\partial n_{1 f}^{*}}{\partial x}
$$

and

$$
\frac{\partial^{2} n_{1 f}}{\partial t^{2}}+v \frac{\partial n_{1 f}}{\partial t}+\omega_{p}^{2} n_{1 f}-\frac{n_{0} r k^{2}}{\varepsilon} \bar{E} u^{*}=-\bar{E} \frac{\partial n_{1 s}^{*}}{\partial z} .
$$

Equations (8) exhibit the coupling between the slow and the fast components of density perturbation via the pump electric field.

We now address ourselves to the theoretical formulation of the third-order optical susceptibility. We start with the resonant Stokes' component of the current density arising due to finite nonlinear induced polarization expressed as

$$
J\left(\omega_{1}\right)=n_{0} e v_{1 f}+n_{1 s}^{*} e v_{0} .
$$

The theoretical formulations as developed above can be employed to obtain

$$
\begin{aligned}
J\left(\omega_{1}\right)=\frac{i \varepsilon \omega_{p}^{2} E_{1}\left(\omega_{1}\right)}{\omega_{1}}+\frac{i r^{2} k^{2} \omega_{p}^{2}\left|E_{0}\right|^{2} E_{1}\left(\omega_{1}\right)}{2 \rho \omega_{0}\left(\omega_{a}^{2}-k^{2} v_{a}^{2}-2 i \Gamma_{a} \omega_{a}\right)} \times \\
\times\left[1-\frac{\left(\delta_{1}^{2}-i \omega_{1} v\right)\left(\delta_{2}^{2}+i \omega_{a} v\right)}{k^{2}|\bar{E}|^{2}}\right]^{-1} .
\end{aligned}
$$


Here $\delta_{1}^{2}=\omega_{p}^{2}-\omega_{1}^{2}, \delta_{2}^{2}=\omega_{p}^{2}-\omega_{a}^{2}$ and $v_{a}\left[=(C / \rho)^{\frac{1}{2}}\right]$ is the acoustic velocity in the crystal medium. The first term in $(10)$ represents the linear component of the induced current density. The second term is the first order nonlinear current density and is held responsible for the nonlinear coupling between the three interacting waves. The time integral of (10) yields the expression for the nonlinear polarization $P_{c d}\left(\omega_{1}\right)$ with its origin being in the generation of free carrier plasma in the medium. Following Aghamkar et al (1988), we obtain the nonlinear induced polarization due to the perturbed current density as

$$
\begin{aligned}
& P_{c d}\left(\omega_{1}\right)=\frac{-r^{2} k^{2} \omega_{p}^{2}\left|E_{0}\right|^{2} E_{1}\left(\omega_{1}\right)}{2 \rho \omega_{0} \omega_{1}\left(\omega_{a}^{2}-k^{2} v_{a}^{2}-2 i \Gamma_{a} \omega_{a}\right)} \times \\
& \times\left[1-\frac{\left(\delta_{1}^{2}-i \omega_{1} v\right)\left(\delta_{2}^{2}+i \omega_{a} v\right)}{k^{2}|\bar{E}|^{2}}\right]^{-1}
\end{aligned}
$$

It is well known that the origin of the SBS process lies in that component of $P_{c d}\left(\omega_{1}\right)$ which depends upon $\left|E_{0}\right|^{2} E_{1}$ and the corresponding third-order optical susceptibility is termed as the Brillouin susceptibility $\chi_{\mathrm{B}}$. The above condition enables one to establish the threshold nature for the onset of SBS. The interaction between the pump and the centrosymmetric crystal will be dominated by the stimulated Brillouin scattering phenomena at a pump power level well above the threshold condition given by

$$
\left|E_{o t h}\right|^{2}=\frac{m^{2}}{e^{2} k^{2}}\left(\omega_{p}^{2}-\omega_{1}^{2}-i \omega_{1} v\right)\left(\omega_{p}^{2}-\omega_{a}^{2}+i \omega_{a} v\right)
$$

Equation (12) has been obtained by setting $P_{c d}\left(\omega_{1}\right)=0$ in (11) which can be treated as the onset condition for the nonlinear scattering phenomena. The corresponding excitation intensity $I_{\text {oth }}$ can be calculated by using the well-known relation

$$
I_{o t h}=\frac{1}{2} \eta_{0} \varepsilon_{0} c\left|E_{o t h}\right|^{2}
$$

where $\eta_{0}, \varepsilon_{0}$ and $c$ are the crystal background refractive index, absolute permittivity and absolute velocity of light, respectively.

At a pump intensity well above the threshold, (11) can be used to obtain the Brillouin susceptibility as

$$
\chi_{\mathrm{B}}=\frac{-r^{2} k^{2} \omega_{p}^{2}}{2 \varepsilon_{0} \rho \omega_{0} \omega_{1}\left(\omega_{a}^{2}-k^{2} v_{a}^{2}-2 i \Gamma_{a} \omega_{a}\right)} .
$$

From (13), one may find that $\chi_{B}$ depends upon material parameters, in particular, the equilibrium carrier concentration $n_{0}$ via the electron plasma frequency $\omega_{p}$. Consequently, we concentrate on an analytical investigation of the Brillouin gain mechanisms in doped semiconductors with both low and high values of $n_{0}$ to cover the entire infrared spectrum.

In doing so, we consider the electrostrictive polarization which can be derived from (5) as

$$
P_{e s}\left(\omega_{1}\right)=\frac{-r^{2} k^{2}\left|E_{0}\right|^{2} E_{1}\left(\omega_{1}\right)}{2 \rho\left(\omega_{a}^{2}-k^{2} v_{a}^{2}-2 i \omega_{a} \Gamma_{a}\right)} \equiv \varepsilon_{0} \chi_{\mathrm{B}, e s}\left|E_{0}\right|^{2} E_{1}\left(\omega_{1}\right)
$$


The above equation together with (11) reveals that the effective induced nonlinear polarization (proportional to $\left|E_{0}\right|^{2} \cdot E_{1}$ in doped centrosymmetric crystal) arises due to both free carrier plasma generation and finite electrostrictive coupling. Thus, one may obtain

$$
P_{n l, \text { eff }}\left(\omega_{1}\right)=P_{c d}\left(\omega_{1}\right)+P_{e s}\left(\omega_{1}\right)
$$

We may define the effective Brillouin susceptibility through the relation

yielding

$$
P_{n l, \text { eff }}=\varepsilon_{0} \chi_{\mathrm{B}, \text { eff }}\left|E_{0}\right|^{2} E_{1}
$$

$$
\chi_{\mathrm{B}, \mathrm{eff}}=\frac{-r^{2} k^{2}}{2 \rho \varepsilon_{0}\left(\omega_{a}^{2}-k^{2} v_{a}^{2}-2 i \omega_{a} \Gamma_{a}\right)}\left[1+\frac{\omega_{p}^{2}}{\omega_{0} \omega_{1}}\right] .
$$

It is clear from the above expressions that significant enhancement of the Brillouin susceptibility is possible under the condition $\omega_{p}^{2} \gg \omega_{0} \omega_{1}$. In other words, appreciable $n$-type doping of the semiconducting crystal can be fruitful in achieving larger Brillouin susceptibility and consequent gain constant. In order to obtain the effective Brillouin gain constant $\left(g_{\mathrm{B}}\right)_{\mathrm{eff}}$, one may use the relation (Yariv 1975)

$$
g_{\mathrm{B}}=-\frac{k}{2 \varepsilon_{i}} \chi_{\mathrm{Bi}}\left|E_{0}\right|^{2}
$$

where we have taken $\chi_{\mathrm{B}}=\chi_{\mathrm{Br}}+i \chi_{\mathrm{B} i}$.

Substituting the value of $\chi_{\mathrm{B} i \text {, eff }}$ from (16), we get

$$
\left(g_{\mathrm{B}}\right)_{\mathrm{eff}}=\frac{r^{2} k^{3}}{8 \varepsilon_{0} \varepsilon_{l} \rho \omega_{a} \Gamma_{a}}\left[1+\frac{\omega_{p}^{2}}{\omega_{0} \omega_{1}}\right]\left|E_{0}\right|^{2} .
$$

Equation (18) has been derived under the situation of almost dispersion-less acoustic wave propagation in the crystal (i.e. $\omega_{a}^{2} \simeq k^{2} v_{a}^{2}$ ).

\section{Results and discussions}

The forthcoming analysis is devoted to the study of the Brillouin gain constant in semiconductors over a wide range of doping (from low to reasonably high). For lightly and heavily doped semiconductors, we may consider $\omega_{0}\left(\sim \omega_{1}\right) \gg \omega_{p} \sim \omega_{a} \sim v$ and $\omega_{0}\left(\sim \omega_{1}\right) \simeq \omega_{p} \gg \omega_{a} \simeq v$, respectively. Under both the above regimes, the threshold value of the pump electric field for the onset of SBS can be obtained from (12) as

$$
\begin{aligned}
& \left(E_{o t h}\right)_{l}>\frac{m}{e k} v\left(\omega_{1} \omega_{a}\right)^{1 / 2}, \\
& \left(E_{o t h}\right)_{h}>\frac{m}{e k} v^{1 / 2} \omega_{p h}^{3 / 2},
\end{aligned}
$$

where the suffixes $l$ and $h$ stand for 'low' and 'high' doping regimes, respectively. The pump electric field amplitude $E_{0}$ should be larger than $\left(E_{o t h}\right)_{l, h}$ for the growth of the backward scattered Brillouin mode. Well above the threshold pump amplitude, the 
expression for the gain constant is

$$
\left[\left(g_{\mathrm{B}}\right)_{\mathrm{eff}}\right]_{l, h}=\frac{r^{2} k^{3}}{8 \varepsilon_{0} \varepsilon_{l} \rho \Gamma_{a} \omega_{a}}\left[1+\frac{\omega_{p l, h}^{2}}{\omega_{0} \omega_{1}}\right]\left|E_{0}\right|_{l, h}^{2} .
$$

It is evident from (20) that at $\omega_{p}^{2} \gg \omega_{0} \omega_{1}$, the nonlinear induced polarization due to nonlinear current density becomes more significant than the one with electrostrictive mechanism as its origin.

The analysis of the SBS threshold field manifests that the role of doping becomes nontrivial only when the semiconductor-radiation interaction is considerable in the doping regime, satisfying the condition $\omega_{p} \sim \omega_{0}$ since $\omega_{1} \sim \omega_{0}$ for $\omega_{a} \ll \omega_{0}$. We now apply the analysis to the specific case of a nearly centrosymmetric, doped semiconducting crystal, viz., $n$-type InSb at $77 \mathrm{~K}$, under both low and high doping regimes. The material constants are taken as $m=0.014 m_{0}, \varepsilon_{l}=15.8, r=5 \times 10^{-10} \mathrm{Fm}^{-1}, \rho=5.8 \times 10^{3} \mathrm{kgm}^{-3}$, $k=5 \times 10^{7} \mathrm{~m}^{-1}, \quad v=3 \times 10^{11} \mathrm{~s}^{-1}, \omega_{0}=1.78 \times 10^{14} \mathrm{~s}^{-1}, \omega_{a}=2 \times 10^{11} \mathrm{~s}^{-1}, \omega_{1}=$ $1.778 \times 10^{14} \mathrm{~s}^{-1}$ and $\Gamma_{a}=2 \times 10^{10} \mathrm{~s}^{-1}$.

For a low doped semiconductor, we take a particular value of $n_{0}=2 \times 10^{19} \mathrm{~m}^{-3}$ in an $n$-type crystal and find from (9a) that the threshold of electric field amplitude required for the onset of SBS is around $3 \times 10^{3} \mathrm{Vm}^{-1}\left(\right.$ i.e. $4.75 \times 10^{8} \mathrm{Wcm}^{-2}$ ) with the corresponding value of the Brillouin susceptibility $\left(\chi_{B}\right)_{l}$ estimated to be $4 \times 66 \times 10^{-16}$ esu obtainable from (13) for $\omega_{p}^{2} \ll \omega_{0} \omega_{1}$. Since this value of $\left(\chi_{B}\right)_{l}$ is very small, we have shifted our interest from the low doping to the high doping regime.

In order to study the detailed behaviour of the SBS threshold condition under the heavy doping regime, we have made use of $(19 b)$ and plotted $\left(E_{o t h}\right)_{h}$ as a function of the doping concentration $n_{0}$ in figure 1 . It can be seen from this figure that $E_{o t h}$

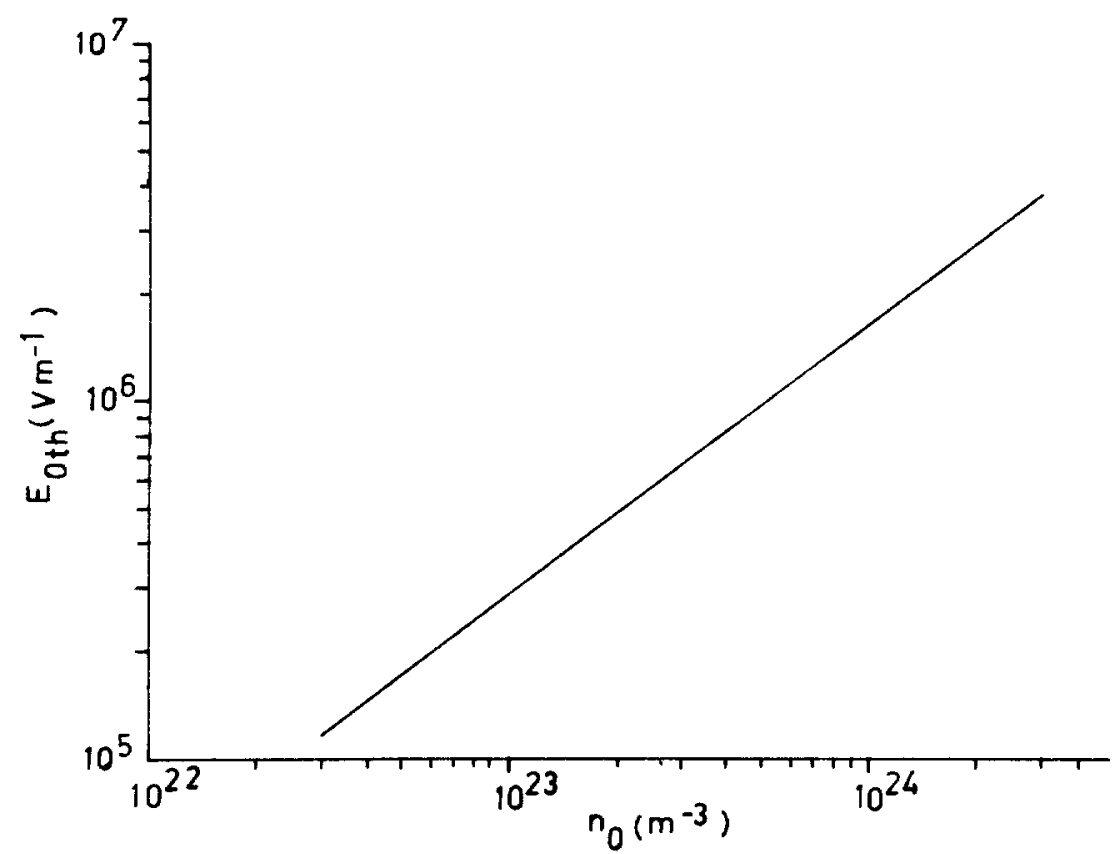

Figure 1. The variation of the threshold pump electric field $E_{\text {oth }}$ with carrier concentration $n_{0}$. Here, $k=5 \times 10^{7} \mathrm{~m}^{-1}$ and $\omega_{0}=1.78 \times 10^{14} \mathrm{~s}^{-1}$. 


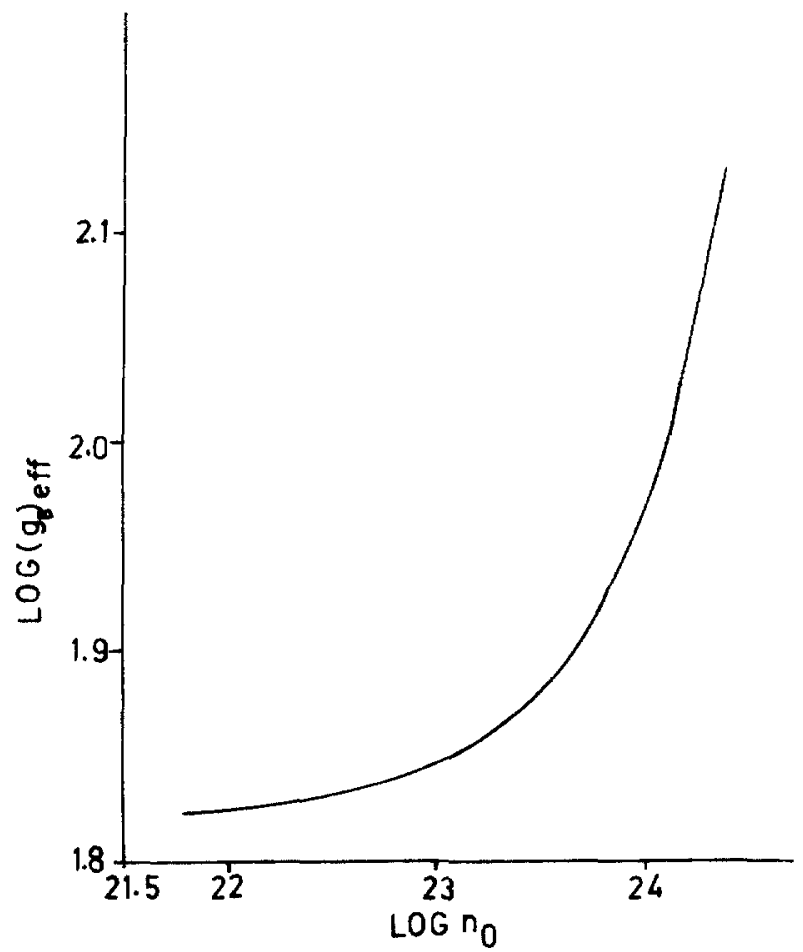

Figure 2. Dependence of the effective Brillouin gain constant $\left(g_{\mathrm{B}}\right)_{\text {eff }}$ on the carrier concentration $n_{0}$ at $E_{0}=1.615 \times 10^{7} \mathrm{Vm}^{-1}$ and $\omega_{0}=1.78 \times 10^{14} \mathrm{~s}^{-1}$.

increases linearly with the increase in $n_{0}$. To estimate the Brillouin susceptibility under a high doping regime $\left(\chi_{B}\right)_{h}$, we made a representative calculation using (13) for $n_{0}=2.44 \times 10^{24} \mathrm{~m}^{-3}$ and found $\left(\chi_{\mathrm{B}}\right)_{h} \sim 5.455 \times 10^{-11} \mathrm{esu}$, which is about 5 orders higher than that under the low doping regime while the Brillouin susceptibility with its origin in the electrostrictive coupling mechanisms is found from (14) to be around $5.43 \times 10^{-11}$ esu.

The above results indicate that significant gain can be achieved when one deals with highly doped semiconductors. Accordingly, we have studied the nature of variation of the effective Brillouin gain constant as a function of doping level in figure 2 for a constant pump amplitude $E_{0}=1.615 \times 10^{7} \mathrm{Vm}^{-1}$ corresponding to $I_{0}=27.5 \mathrm{MW} \mathrm{cm}^{-2}$. It exhibits an almost doping-independent behaviour of the Brillouin gain constant till $\omega_{p}^{2}$ becomes comparable to $\omega_{0} \omega_{1}$. On further increase in the doping level, $\left(g_{\mathrm{B}}\right)_{\text {eff }}$ increases sharply. This result leads one to the conclusion that a large Brillouin gain constant can be achieved in highly doped semiconducting crystals. It may be recalled here that doping cannot be increased arbitrarily in semiconductors. Moreover, the SBS threshold increases with the doping level. Hence one has to select an appropriate doping concentration as well as a pump electric field amplitude, well below the damage threshold, to achieve large gains in the backscattered Stokes' components.

In order to employ the theoretical formulations to explore the possibility of occurrence of optical phase conjugation via stimulated Brillouin scattering (OPC-SBS) in nearly centrosymmetric crystals, we now consider the OPC threshold condition 
which is given as $\left[\left(g_{\mathrm{B}}\right)_{\text {eff }}\right] L \simeq 30$ (Zel'dovich et al 1985), $L$ being the cell length. It may be noted here that the gain constant arising only due to the electrostrictive coupling in the present formulation, obtainable from eq. (18) by setting $\omega_{p}=0$, is identical to that of Zel'dovich et al [1985, equation (2.1.11)]. Thus one can achieve OPC-SBS in $n$-InSb if the cell length $(L)$ is taken $\sim 30 /\left[\left(g_{\mathrm{B}}\right)_{\text {eff }}\right]$; for $L \approx 0.24 \mathrm{~m}$ and $n_{0}=2.44 \times 10^{24} \mathrm{~m}^{-3}, I_{0} \simeq 27.5 \mathrm{MW} \mathrm{cm}^{-2}$ and $\left[\left(g_{\mathrm{B}}\right)_{\mathrm{eff}}\right] \simeq 125 \mathrm{~m}^{-1}$. One can reduce the cell length further by increasing the doping level as well as excitation intensity taking care that the crystal does not undergo any damage.

From the above discussions, it is clear that highly doped nearly centrosymmetric crystals like $n$-GaAs, $n$-InSb, etc. could be a class of materials suitable for the achievement of high purity efficient optical phase conjugation via stimulated Brillouin scattering mechanisms.

\section{Acknowledgements}

The authors are indebted to Dr P Sen for constant encouragement. Financial assistance from the Department of Science and Technology is gratefully acknowledged.

\section{References}

Aghamkar P, Sen P and Sen P K 1988 Phys. Status Solidi 145343

Hayes W and Loudon R 1978 Scattering of light by crystals (New York: Wiley) p. 301

Jain R K 1982 Opt. Eng. 21199

Mahan G D 1981 Many-particle physics (New York: Plenum) p. 543

Sen P and Sen P K 1985 Phys. Rev. B31 1034

Sen P and Sen P K 1986 Phys. Rev. B33 1427

Shen Y R and Bloembergen N 1965 Phys. Rev. 137 A1787

Smith S D 1985 Nature (London) 316 319, and references therein

Yariv A 1975 Quantum electronics 2nd edn (New York: Wiley) p. 491

Zel'dovich B Y, Pilipetsky N F and Shkunov V V 1985 Principles of phase conjugation (Berlin: Springer-Verlag) 